\title{
Function and Structure of the Right Inferior Frontal Cortex Predict Individual Differences in Response Inhibition: A Model-Based Approach
}

\author{
Birte U. Forstmann, ${ }^{1}$ Sara Jahfari, ${ }^{1}$ H. Steven Scholte, ${ }^{2}$ Uta Wolfensteller, ${ }^{3}$ Wery P. M. van den Wildenberg, ${ }^{1}$ and \\ K. Richard Ridderinkhof ${ }^{1}$ \\ Departments of ${ }^{1}$ Psychology, Amsterdam Center for the Study of Adaptive Control in Brain and Behavior (Acacia) and ${ }^{2}$ Psychology, University of \\ Amsterdam, 1018 WB, Amsterdam, the Netherlands, and ${ }^{3}$ Department of Cognitive Neurology, Max Planck Institute for Human Cognitive and Brain \\ Sciences, 04103 Leipzig, Germany
}

The ability to suppress one's impulses and actions constitutes a fundamental mechanism of cognitive control, thought to be subserved by the right inferior frontal cortex (rIFC). The neural bases of more selective inhibitory control when selecting between two actions have thus far remained articulated with less precision. Selective inhibition can be explored in detail by extracting parameters from response time (RT) distributions as derived from performance in the Simon task. Individual differences in RT distribution parameters not only can be used to probe the efficiency and temporal dynamics of selective response inhibition, but also allow a more detailed analysis of functional neuroimaging data. Such model-based analyses, which capitalize on individual differences, have demonstrated that selective response inhibition is subserved by the rIFC. The aim of the present study was to specify the relationship between model parameters of response inhibition and their functional and structural underpinnings in the brain. Functional magnetic resonance imaging (fMRI) data were obtained from healthy participants while performing a Simon task in which irrelevant information can activate incorrect responses that should be selectively inhibited in favor of selecting the correct response. In addition, structural data on the density of coherency of white matter tracts were obtained using diffusion tensor imaging (DTI). The analyses aimed at quantifying the extent to which RT distribution measures of response inhibition are associated with individual differences in both rIFC function and structure. The results revealed a strong correlation between the model parameters and both fMRI and DTI characteristics of the rIFC. In general, our results reveal that individual differences in inhibition are accompanied by differences in both brain function and structure.

Key words: inhibition; interference control; model-driven fMRI; DTI; RT distribution analysis; cognition

\section{Introduction}

The aim of the present investigation is to bring to light the neural bases and dynamics of inhibitory control processes during response selection using model-driven functional magnetic resonance imaging (fMRI) and diffusion tensor imaging (DTI). Model-driven fMRI refers to the combination of fMRI with specific model-based parameters that reflect individual differences in the efficiency of specific cognitive processes (compare Daw et al., 2006). This approach has been demonstrated to provide novel insights into individual differences in neurocognitive processes involved in reward-based decision-making (Daw et al., 2006; Haruno and Kawato, 2006) and in the ability to inhibit ongoing responses (Aron and Poldrack, 2006). DTI analyses (which allow the study of structural connectivity by quantifying the density of

\footnotetext{
Received April 5, 2008; revised July 25, 2008; accepted Aug. 18, 2008.

This work was supported by a Vici grant (B.U.F. and K.R.R.) and a Veni grant (W.P.M.vdW.) from the Netherlands Organization for Scientific Research (NWO). We thank Matthew Rushworth for valuable comments.

Correspondence should be addressed to Birte U. Forstmann, University of Amsterdam, Department of Psychology, Amsterdam Center for the Study of Adaptive Control in Brain and Behavior (Acacia), Roetersstraat 15, 1018 WB Amsterdam, the Netherlands, E-mail: b.u.forstmann@uva.nl.

D0I:10.1523/JNEUROSCI.1465-08.2008

Copyright $\odot 2008$ Society for Neuroscience $\quad 0270-6474 / 08 / 289790-07 \$ 15.00 / 0$
}

white matter tracts) revealed that the right inferior frontal cortex (rIFC) is tightly connected with the pre-supplementary motor area (SMA) and subthalamic nucleus (Aron et al., 2007), indicating a brain network associated with stopping a motor response (i.e., nonselective response inhibition). Recently, parameters derived from response time (RT) distribution analysis techniques have been used in model-based fMRI to examine individual differences in the ability to selectively inhibit responses during response selection (Forstmann et al., 2008). "Selective response inhibition" refers descriptively to action selection mechanisms that serve to reduce interference between competing actions through inhibition of incorrect response impulses. Selective response inhibition is a key aspect of cognitive control that has been studied extensively with classical conflict tasks as the Stroop (1935), the Eriksen (Eriksen and Eriksen, 1974), and the Simon (Simon, 1967) task.

Dual-route architectures, proposed to capture the temporal dynamics of information processing while performing on conflict tasks (Kornblum et al., 1990; Eimer, 1995), have been extended to include selective suppression of response-related activation when two responses are activated simultaneously (Ridderinkhof, 2002). To account for the temporal dynamics of 


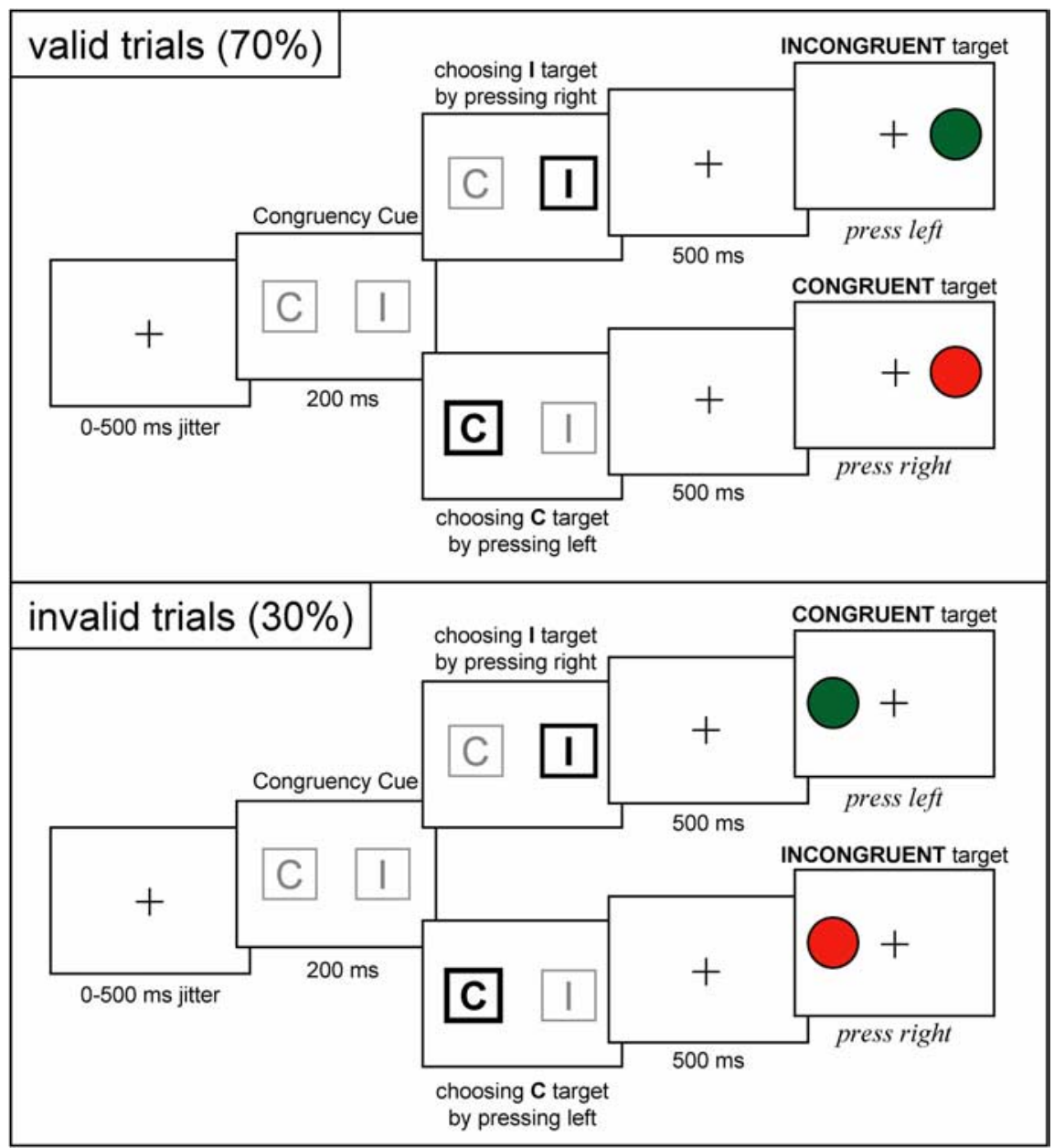

Figure 1. Schematic drawing of the modified Simon task. Each trial started with the presentation of a congruency cue. In the choice condition, participants indicated their preference for receiving a congruent or incongruent target stimulus by selecting either C (congruent) or I (incongruent) with the middle finger or the index finger of the left hand. In the no-choice condition (data not shown here), the congruency cue either conveyed C or I information. The target matched the cue in $70 \%$ of the trials (valid trials; top), whereas in $30 \%$ of the trials, target congruence did not match the cue (invalid trials; bottom). The target stimuli consisted of green and red circles that were associated with a left and right button press, respectively, with the index or middle fingers of the right hand. In congruent trials, responses were spatially compatible with the location of the target stimulus (e.g., left green circle leads to an index finger response), whereas in incongruent targets, participants' responses were spatially incompatible with the location of the stimulus (e.g., left red circle leads to a middle finger response).

selective response inhibition and their covariation with brain function and structure, RT distribution analyses can be used. More specifically, to quantify individual differences in selective inhibition, delta plots can be constructed by plotting the interference effect as a function of response speed (de Jong et al., 1994; Ridderinkhof, 2002). In accordance with the dual-route architecture, delta plots reveal that interference effects in conflict tasks are reduced for relatively slow responses, and more so for participants who are proficient in inhibition.

We studied selective response inhibition in the Simon task by capitalizing on individual differences in RT distribution parameters. In the Simon task, the task-irrelevant spatial position of the stimulus can capture a response that is either congruent or incongruent with the response designated by the color of the stimulus. To manipulate the strength of this congruency effect, participants were cued (either validly or invalidly) about the congruency of the upcoming stimulus. Invalidly (compared with validly) cued stimuli are associated with stronger inhibition to reduce the unexpected response conflict.

We formulate three hypotheses. First, we expect rIFC blood oxygenation level-dependent (BOLD) activation to covary with proficiency in implementing selective response inhibition as reflected by the individual RT distribution parameters. Second, compared with less proficient inhibitors, those participants who are more proficient in selective suppression show increased structural connectivity in the rIFC as measured by fractional anisotropy (FA), reflecting the density of coherent white matter tracts. Third, rIFC BOLD activation and rIFC local structural connectivity correlate positively, reflecting a tight linkage between brain function and structure in explaining individual differences in cognitive control.

\section{Materials and Methods}

Participants. Seventeen healthy volunteers were recruited. We obtained written consent from all participants before the scanning session. The experiment was approved by the local ethics committee of the University of Amsterdam, and all procedures were conducted in accordance with relevant laws and institutional guidelines. All subjects had normal or corrected-to-normal vision. No subject had a history of neurological, major medical, or psychiatric disorder, according to self-report. The data of three subjects were excluded from the fMRI analysis because of excessive movement artifacts as well as a technical problem with the response system. The remaining 14 subjects were 11 females and 3 males (mean age, 25 years; SD, 2.8 years), who were all right-handed as assessed by the Edinburgh Inventory (Oldfield, 1971). For the DTI analysis, only 11 of the $14 \mathrm{fMRI}$ participants were included because of technical problems during the DTI acquisition. However, to increase statistical power, we included two additional subjects who were excluded from the fMRI analysis because of movement artifacts. The remaining 13 subjects were 9 females and 4 males (mean age, 26 years; $\mathrm{SD}, 3.2$ years).

Behavioral task. We used a cueing version of the Simon task (Simon, 1967) (Fig. 1). The Simon target stimuli consisted of green or red circles presented either to the left or to the right of a central fixation cross. Participants were instructed to press the left button to green stimuli and a right button to red stimuli, respectively, with the index and middle fingers of the right hand. Stimulus color (green vs red) is the relevant aspect of the signal, whereas spatial location (left vs right) is the irrelevant dimension. On congruent trials, the responses are spatially compatible with the position of the target (e.g., a green circle designating a left button press and appearing left of fixation). On incongruent trials, the responses are spatially incompatible with the target location (e.g., a red circle designating a right response but appearing left). The distance between the fixation cross and the stimulus covered a visual angle of $2.8^{\circ}$.

On each trial, presentation of the colored circle was preceded by the presentation of a congruency cue. In the choice condition, participants had to choose between receiving a congruent versus an incongruent target signal. The congruency cue looked like "[C I]," and pressing the left response button meant opting for a congruent target, whereas pressing right marked preferring an incongruent target signal. The left and right buttons for responding to the congruency cue were operated, respectively, with the middle and index fingers of the left hand. Follow-up 
analyses indicated that participants selected "C" and "I" cues equally often (i.e., frequencies did not differ from $50 \%, t<1$ ). On no-choice trials, subjects could not choose target congruency and were just presented with a "C" or an "I" cue. Nevertheless, participants also had to press left or right to no-choice cues to ensure that responding to cues was counterbalanced across choice and no-choice trials.

The design also included a cue validity manipulation. On $70 \%$ of the trials, called "valid trials," target congruency matched the cue. For example, the participant selected a "C" cue (choice condition) or was presented with a " $\mathrm{C}$ " cue (no-choice condition) and did receive a congruent target. Importantly, on $30 \%$ of the trials, target congruency did not match the congruency information conveyed by the cue (e.g., a "C" cue was followed by an incongruent target). This category of trials will be referred to as "invalid trials."

All stimuli were presented on a back-projection screen that was viewed via a mirror system attached to the magnetic resonance imaging (MRI) head coil. Participants practiced the relevant mapping in a short training session of $\sim 10$ min before entering the MRI scanner.

The timing of the sequence of trials was triggered from the MRI control every $6 \mathrm{~s}$. The trials started with a variable oversampling interval of 0 , 500,1000 , or $1500 \mathrm{~ms}$ to obtain an interpolated temporal resolution of $500 \mathrm{~ms}$. A fixation cross was presented during the variable oversampling interval. Participants were asked to maintain fixation. The congruency cue was presented for $2000 \mathrm{~ms}$ in the middle of the screen, and participants selected one of the cues using their left index or middle finger. After the cue presentation, a short fixation interval of $500 \mathrm{~ms}$ was presented. Next, the target stimulus was displayed until the response interval exceeded $1500 \mathrm{~ms}$ while participants had to respond with their right index or middle finger. Finally, feedback was presented for $350 \mathrm{~ms}$ indicating a correct response, an incorrect answer, or miss. Because of varying response times and initial oversampling intervals, the intertrial interval ranges between 650 and $3150 \mathrm{~ms}$.

The experiment consisted of 380 trials including 20 null events that were pseudo-randomly interspersed. The null events were included to compensate the overlap of the BOLD response between adjacent trials. The functional scanning lasted $\sim 40 \mathrm{~min}$, separated in two experimental blocks. Every block started with two dummy trials that were excluded from the analysis.

$R T$ distribution analysis of interference effects. We used the dual-process model of interference effects in the Simon task and used the associated RT distributional analyses to quantify the effects of selective response inhibition to examine which brain areas covary in activation with individual differences in the response inhibition parameter.

In accordance with this general dual-route architecture, a recurrent and critical observation is that fast responses to incongruent stimuli tend to be associated with below-chance accuracy (Gratton et al., 1992), which has been referred to as response capture. To overcome direct response capture by task-irrelevant information, many authors have assumed implicitly or explicitly that the response as activated by irrelevant stimulus features is subsequently inhibited (Eimer, 1999) and aborted (Logan and Cowan, 1984). Such inhibition is not operational instantaneously but requires some time to develop (Eimer, 1999) during target processing. Because of these dynamics (the gradual build-up of response inhibition as time progresses across a trial), slower responses will benefit more from selective response inhibition than faster responses (Burle et al., 2002; Eimer, 1999; Wylie et al., 2007). Whereas fast responses are dominated by direct location-driven response capture, an additional factor comes into play for slower responses: with slower responses, the selective inhibition process has had time to develop, and thus the activation of the incorrect response along the direct capture route will be reduced. Consequently, correct slow responses to congruent stimuli will be less facilitated by the position-driven response capture, whereas correct slow responses to incongruent stimuli will be less delayed. Thus, given these dynamics, interference effects are affected by selective response inhibition more in slow than in fast responses (for review, see Ridderinkhof et al., 2005). These dynamics are well captured by so-called delta plots, constructed by plotting the interference effect as a function of response speed (de Jong et al., 1994; Ridderinkhof, 2002; Wiegand and Wascher, 2007). Whereas delta plots prototypically have a positive slope (i.e., the effects of any experi- mental factor increase as a function of response speed), the notion that selective inhibition results in a reduction of the interference effect in slow responses (outlined above) implies a different delta-plot pattern: here the interference effect does not increase linearly as a function of response speed, but instead levels off and reduces for slow responses. If more effective selective inhibition results in a more pronounced reduction of interference effects in slow responses, as argued above, then the leveling off of the delta plot should be more pronounced in individuals who are more proficient in response inhibition than in less proficient individuals. Moreover, this reduction should be stronger on invalidly cued trials compared with trials in which cue and target congruency matched. Thus, the slope of the slowest portion of the delta plot may serve as a parametric index sensitive to individual differences in selective response inhibition.

Magnetic resonance imaging scanning procedure. The DTI and fMRI measurements were acquired in a single scanning session on a 3T scanner (Philips). For the fMRI experiment, 30 axial slices were acquired $\left(222^{2}\right.$ $\mathrm{mm}$ FOV , $96 \times 96$ in plane resolution, $3 \mathrm{~mm}$ slice thickness, $0.3 \mathrm{~mm}$ slice spacing) parallel to the anterior cingulate-posterior cingulate plane and covering the whole brain. We used a single-shot, gradient-recalled echo planar imaging sequence (repetition time $2000 \mathrm{~ms}$, echo time $28 \mathrm{~ms}, 90^{\circ}$ flip-angle, transversal orientation). Before the functional runs, a threedimensional (3D) T1 scan was acquired [T1 turbo field echo, $25^{2} \mathrm{~cm}$ FOV $256^{2}$ in plane resolution, 182 slices, slice thickness 1.2 , repetition time (TR) 9.69, echo time (TE) 4.6, FA 8, sagittal orientation].

The diffusion-weighted images (DWIs) (TR $7720 \mathrm{~ms}$, TE $94 \mathrm{~ms}$, flip angle $90^{\circ}$, FOV $224 \times 224 \mathrm{~mm}$, matrix size $128 \times 128,40$ slices, $b=600$, $94 \mathrm{~ms}$ ) were measured after each of the two experimental blocks in 32 non-collinear directions. The end of each series of directions was preceded by acquisition of a non-diffusion-weighted volume for purposes of registration for motion correction. Each DWI acquisition phase lasted for $5.2 \mathrm{~min}$.

Functional magnetic resonance analysis. Analysis was performed using FEAT (FMRI Expert Analysis Tool) Version 4.0, part of FSL (FMRIB's Software Library, www.fmrib.ox.ac.uk/fsl). The first two volumes were discarded to allow for $\mathrm{T} 1$ equilibrium effects. The remaining images were then realigned to compensate for small head movements (Jenkinson et al., 2002). Data were spatially smoothed using a $5 \mathrm{~mm}$ full-width-halfmaximum Gaussian kernel. The data were filtered in the temporal domain using a high-pass filter with a cutoff frequency of $1 / 50 \mathrm{~Hz}$ to correct for baseline drifts in the signal. Finally, the functional data were prewhitened using FSL (FMRIB's Software Library, www.fmrib.ox.ac.uk/ fsl) (Woolrich et al., 2001).

All functional data sets were individually registered into 3D space using the participants' individual high-resolution anatomical images acquired at the beginning of each scanning session. The individual 3D reference data set was used to normalize the functional data into Montreal Neurological Institute (MNI) space by linear scaling (affine transformations) (Jenkinson and Smith, 2001). The statistical evaluation was performed using the general linear model. The design matrix was generated with a synthetic hemodynamic response function and its first derivative. Individual contrast maps were generated for incongruent compared with congruent trials, separately for cue validity (70\% valid, 30\% invalid trials). Higher-level analysis was performed using FLAME (FMRIB's Local Analysis of Mixed Effects) (Beckmann et al., 2003; Woolrich et al., 2004). For the whole-brain analysis of incongruent versus congruent trials per cue validity condition as well as the whole-brain covariance analyses, we only report cortical regions with a height threshold of $z>2.3$ and a cluster probability of $p<0.05$, corrected for whole-brain multiple comparisons [using Gaussian random field theory (GRFT)]. For the whole-brain group analysis for good inhibitors versus poor inhibitors, a height threshold of $z>1.9$ and a cluster probability of $p<0.05$, corrected for whole-brain multiple comparisons (GRFT), was used.

The aim of the covariance analyses was to test for individual differences in selective response inhibition (Forstmann et al., 2008). To this end, the individual congruency contrasts (incongruent vs congruent) for the two validity conditions were subjected to two separate covariate analyses to investigate the dynamics of selective response suppression. For each participant, the slope values for the different delta-plot segments (see also Results, Behavior) were included as individual covariates. 


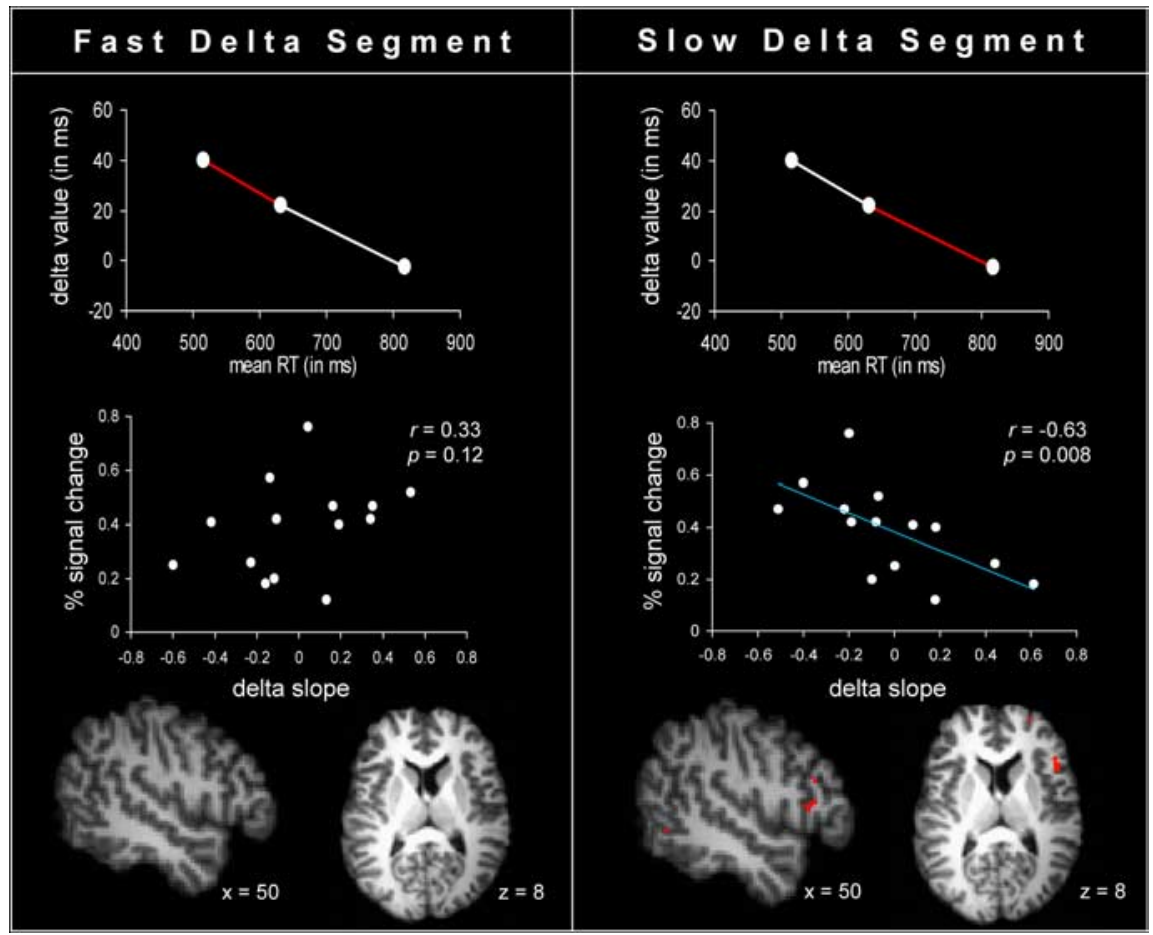

Figure 2. Selective response inhibition covariate analysis with individual parameters derived from 14 participants for each segment of the delta plot (depicted in red). Top, The delta slopes separated in fast and slow segments for invalidly cued trials. Middle, Pearson correlations (two-tailed) between the percentage of signal changes ( $y$-axis) derived from the IIFC and the demeaned delta slopes ( $x$-axis) for each segment of the delta plot. Bottom, The averaged activation across 14 participants rendered onto a template brain $(z>2.3, p<0.05$, whole-brain corrected) of the covariance analysis with the two segments of the delta plot. The only significant prefrontal activation is obtained for the slowest segment of the delta plot in the rIFC (Brodma$n n ' s$ area $44 ; x=50, y=26, z=8$ ). Coordinates are given in MNI space.

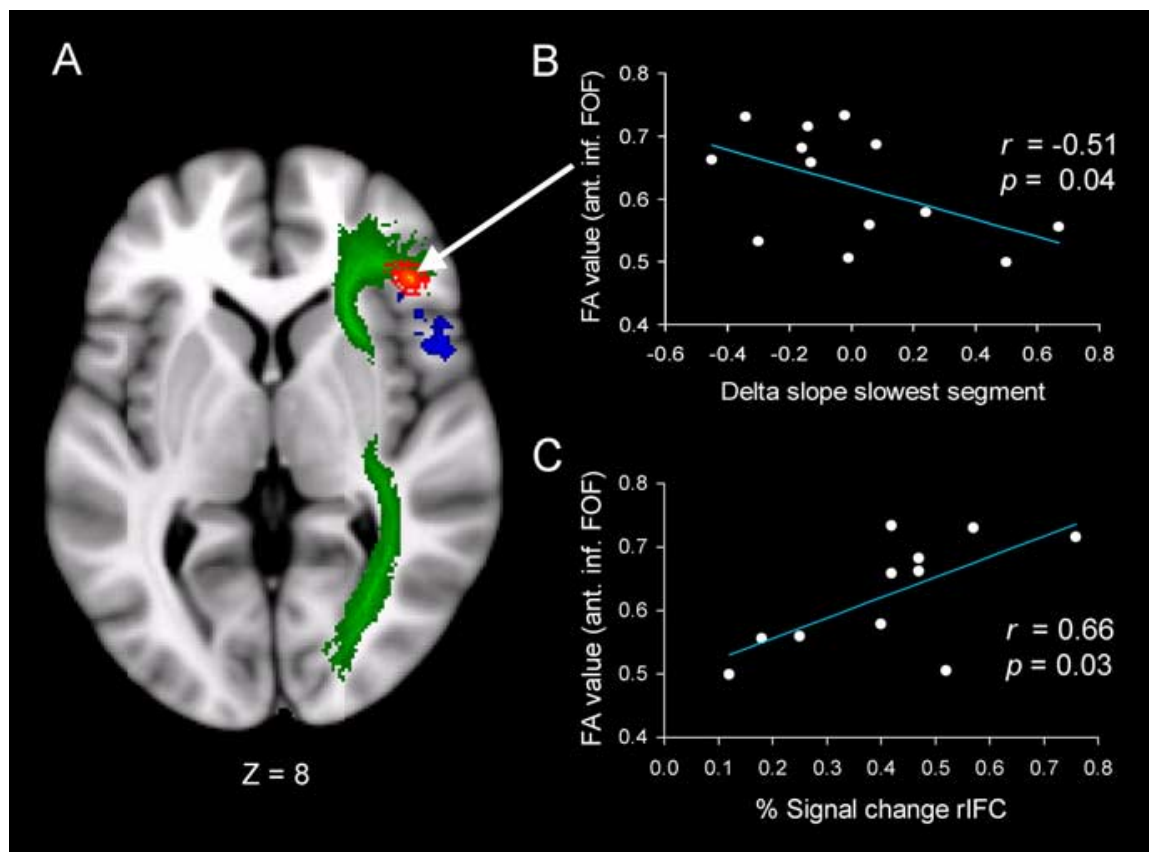

Figure 3. Function and structure of the IIFC. BOLD data were transformed on the basis of a nonlinear transformation from MNI to tract-based space (the transformation used in DTI analysis) to allow comparison of DTI (red) and fMRI (blue) results (see also Materials and Methods). $A$, Axial view. Inferior FOF is displayed in green and derived from the Johns Hopkins University WhiteMatter Tractography Atlas implemented in FSL (FMRIB's Software Library, www.fmrib.ox.ac.uk/fsl; Version 4.0). B, Pearson correlation (one-tailed) between $F A$ values derived from the anterior part of the $\mathrm{FO} F$ and demeaned slope values derived from the delta plot of the slowest segment for invalidly cued trials. C, Pearson correlation (two-tailed) between FA values from the anterior part of the inferior FOF and percentage BOLD signal change derived from the rIFC.
Finally, to verify the functional significance of this analysis, the individual effect size of the overall Simon interference effect was also entered separately as a covariate in the fMRI regression model.

To compute the percentage signal change of the hemodynamic response of the rIFC, all voxels of the relevant contrast exceeding the critical threshold in the mean $\mathrm{z}$-map were determined. We then extracted the time course of the signal underlying these activated voxels for each participant from the modeled data. The percentage signal change was calculated in relation to the mean signal intensity across all time steps for these voxels. The signal change was averaged separately for incongruent versus congruent trials on valid and incongruent versus congruent trials on invalid trials, beginning with the presentation of the cues for each participant. These values were later used to compute the correlation between the percentage signal change in the rIFC (Forstmann et al., 2008) and (1) the two delta slopes derived from the RT distribution analyses (Figs. 2, 3), (2) the mean RT congruency effect, and (3) the FA values derived from the DTI analysis (Fig. 3).

Diffusion tensor imaging analysis. We calculated fractional anisotropy, which reflects the degree of diffusion anisotropy within a voxel, on the basis of the diffusion weighted images. The degree of diffusion anisotropy in a voxel is determined by microstructural features of the tissue in that particular voxel, including fiber properties (for example, fiber diameter and density) and fiber tract coherence. Data analysis and preprocessing were performed with FSLVersion 4.0 (FMRIB's Software Library, www. fmrib.ox.ac.uk/fsl). Correction for eddy currents and head movement was done by means of affine registration on a reference volume. First, FA images were created by fitting a tensor model to the raw diffusion data using FMRIB's Diffusion Toolbox, and then brain-extracted using Bet extraction tool (Smith, 2002). A larger FA value indicates a distortion of Brownian motion, which signifies the presence of coherent white matter tracts. Values closer to 0 correspond to more isotropic diffusion of water molecules (showing Brownian motion), and absence or little coherence of white matter.

All subjects' FA data were then aligned into a common space using the nonlinear registration Image Registration Took Kit (Rueckert et al., 1999) in combination with TBSS (tract-based spatial statistics) (Smith et al., 2006). Next, the mean FA image was created and thinned to create a mean FA skeleton, which represents the centers of all tracts common to the group. Each subject's aligned FA data were then projected onto this skeleton and the resulting data were fed into voxel-wise cross-subject statistics.

The significance of the comparison between good inhibitors and poor inhibitors was determined by means of permutation testing using randomizations. We considered activation significant at a $t$ value $>3$, with a minimum cluster size of $45 \mathrm{~mm}^{3}$ resulting in significant differences between good and poor inhibitors. In contrast, comparing groups with a low com- 
pared with a high mean interference effect, or randomly assigned groups, did not reveal significant differences in connectivity measures.

The individual FA values derived from the anterior part of the inferior fronto-occipital fasciculus (FOF) were correlated with the two different delta slopes derived from the RT distribution analyses. Finally, to test our third hypothesis regarding the linkage between structure and function in the rIFC, we computed correlations between the BOLD signal change derived from the rIFC and the FA values derived from the anterior part of the inferior FOF.

\section{Results}

The factor choice (choice vs no-choice) did not affect behavioral measures in any way. Because this choice factor is not relevant for present purposes, data were collapsed across choice and nochoice trials to increase trial numbers.

\section{Behavior}

Omnibus repeated measures multivariate ANOVA tested the main effects of target congruency (incongruent vs congruent) and cue validity (valid vs invalid) on error rates and RT.

\section{Error rates}

Overall, incongruent trials yielded more choice errors than congruent trials, respectively, $3.7 \%$ vs $2.7 \%\left(F_{(1,13)}=6.32, p=\right.$ 0.026 ). Error rates did not vary systematically with the (main or interaction) effects of cue validity, $F<1$.

\section{Reaction times}

The factor congruency produced its typical effect on RT, with incongruent responses ( $658 \mathrm{~ms}$ ) being $\sim 28 \mathrm{~ms}$ slower than congruent responses $(630 \mathrm{~ms}), F_{(1,13)}=8.95, p=0.01$. RT on invalidly cued trials $(657 \mathrm{~ms}$ ) was marginally slower than on valid trials (632 ms), $F_{(1,13)}=4.05, p=0.07$. Importantly, the size of the interference effect depended on cue validity, with invalid trials yielding a significantly reduced congruency effect compared with valid trials ( 18 vs $\left.39 \mathrm{~ms}, F_{(1,13)}=4.74, p=0.049\right)$. This was important in establishing that, indeed, responses to incongruent stimuli required the strongest inhibition of the tendency to activate the incorrect response when the subjects in fact expected a congruent target stimulus.

\section{Selective inhibition}

A second set of repeated measures ANOVAs focused on distributional analyses to capture the temporal dynamics of the congruency effect. Overall, the interference effect steadily decreased from 47 to $29 \mathrm{~ms}$ in fast and middle segments, to $11 \mathrm{~ms}$ in the slowest segment, $F_{(2,26)}=5.21, p=0.023$. The delta-plot slopes were generally negative-going ( $p=0.006$ when tested against a zero slope-value), and did not distinguish between valid and invalid trials or between fast and slow RT segments $(F<1, p>$ $0.30)$.

\section{Neuroimaging data \\ fMRI data}

An initial set of conventional analyses was augmented with a model-driven fMRI approach to examine more rigorously the neural underpinnings of the selective response suppression hypothesis. The first set of analyses focused on the main effects of congruency, i.e., incongruent versus congruent trials. Direct comparison of incongruent and congruent trials across cue validity yielded no significant main effect of congruency. The conditional main effect for congruency in the invalid condition revealed only significant activation in the right medial frontal cortex $(4,40,42)$. No significant conditional main effect of target congruency was obtained in the valid cue condition. Thus, in line with earlier findings, the condition that elicited the strongest response capture (when a congruent target was expected but an incongruent target stimulus was delivered) was associated with increased activation in pre-SMA. Comparing good versus poor inhibitors based on a median split using the individual RT distribution parameters (the slope of the slowest segment of the delta plot) in the conditional main effect of congruency for invalidly cued trials revealed significantly stronger activation only in the rIFC $(48,24,8)$ for good compared with poor inhibitors. No significant activation was found in the reverse contrast (i.e., for poor vs good inhibitors).

In the second, model-based, set of analyses we entered individual slope values derived for each segment of the delta plot for validly and invalidly cued trials, respectively, as covariates into the analyses of the conditional main effect of congruency for the two cue validity conditions.

The analyses revealed no significant activations for either the faster or the slower segments in the valid cue condition. In contrast, for the invalidly cued condition, significant prefrontal activation was found only in the $\operatorname{rIFC}(50,26,8)$ for the slowest but not for the fastest segment of the delta plot, a result that was confirmed by a negative correlation between the BOLD signal change derived from the rIFC and the individual slope values of the slowest segment (Fig. 2).

To further verify the functional significance and specificity of the slowest segment of the delta plot as a specific indicator for selective response inhibition, both the overall mean and the individual effect size of the overall interference effect were also entered (separately) as covariates in the fMRI regression model as a control variable. Importantly, these analyses did not yield any significant effects $(F<1)$, confirming that only one specific parameter (i.e., the slope of the slowest segment of the delta plot, specifically predicted individual differences in selective response inhibition) corresponded with rIFC activation.

\section{DTI data}

The aim of the DTI data analysis was threefold. In a first step, we compared FA values from good versus poor inhibitors to test for local structural differences in white matter tracts connecting the rIFC. The results revealed significant connectivity differences for good versus poor inhibitors in the right anterior part of the inferior FOF (Fig. 3A) and in the precuneus. Note that the structural difference found in the anterior part of the inferior FOF might also encompass parts of the uncinate fascicle, the extreme capsule, or the third branch of the superior longitudinal fascicle. Given the spatial resolution of the present data, we cannot reliably distinguish between the different fascicles, for instance, using fiber tracking.

In the next step, FA values derived from the anterior and posterior parts of the inferior FOF were correlated with the slowest segment of the delta plot of the invalidly cued trials. The results revealed a significant negative correlation between the slope values and FA values (Fig. $3 B$ ). This pattern of results resembles the BOLD covariance findings observed for the rIFC in the invalidly cued trials (Fig. 2). As a final step, we tested whether there is a linkage between functional and structural individual differences. Therefore, we computed correlations between the percentage BOLD signal change derived from the rIFC and the FA values derived from the right anterior and posterior part of the inferior FOF. The results revealed a positive correlation between both measures (Fig. 3C). 


\section{Discussion}

In the present study we used a model-driven approach to investigate the functional and structural underpinnings of individual differences of selective response inhibition. A cueing version of the Simon task was used, and the resulting individual RT distribution parameters were incorporated as covariates in the functional imaging regression analysis (Forstmann et al., 2008). Our first hypothesis was that individual RT distribution parameters, reflecting the temporal dynamics of selective response inhibition, predict activation in the rIFC. This hypothesis was confirmed by the present model-based fMRI results (Fig. 2), indicating a tight linkage between function (BOLD activation in rIFC) and behavior (proficiency of selective response inhibition).

Our second hypothesis stated that individuals who are proficient in selective suppression show higher FA connectivity values in white matter tracts in the rIFC than less proficient subjects. To test this conjecture, individual RT distribution parameters were used to classify subgroups of good and poor inhibitors based on a median split of the slowest segment of the delta plots. The connectivity data revealed higher FA values in the right anterior part of the inferior FOF for good versus poor performers, indicating a tight linkage between structure (FA values in FOF) and behavior (proficiency of selective response inhibition). This pattern of the connectivity differences in the anterior part of the FOF closely resembled the corresponding pattern for the BOLD findings for the rIFC.

Finally, our third hypothesis stated that the rIFC BOLD activation and the rIFC local structural differences should correlate positively. This hypothesis was also confirmed by the present data pattern (Fig. 3C), reflecting a systematic linkage of individual selective response inhibition differences at the behavioral, functional, and structural levels, as is supported by independent techniques.

In general, the present results are important because they corroborate and extend recent findings: the role of the rIFC in the implementation of response inhibition (Peterson et al., 2002; Aron and Poldrack, 2006; Aron et al., 2007; Forstmann et al., 2008) is modulated by the validity of congruency cues. In the present study, activation in the rIFC was restricted to invalidly cued incongruent trials when incorporating the slowest segment of the delta-plot slopes into the fMRI regression model. In a comparable analysis for validly cued incongruent trials, no such activation in the rIFC was found. At first sight, this finding might seem controversial since we also observed negative-going deltaplot slopes for the valid cue condition. However, given the reduced interference effect in invalidly cued compared with validly cued trials as well as steeper delta slopes in invalidly cued trials, we argue that inhibition is more strongly required because participants misguidedly prepare for a congruent stimulus and hence rely to some extent on the response capture as triggered by the stimulus location. To overcome this incorrect response capture, stronger inhibition is required compared with validly cued incongruent trials. To validate our results, we incorporated the fastest segment of the delta slopes into the regression analysis for both validly and invalidly cued trials as well as the mean congruency effect and the overall mean RT for each individual. None of these additional analyses yielded significant results, attesting to the specificity of the relation between our measure of response inhibition and rIFC activation.

Next, we were interested in whether the individual covariation of the RT distribution parameters would also be reflected in structural connectivity differences in the rIFC. Therefore, we col- lected DTI data of all participants. In general, there is growing evidence for a direct linkage between BOLD and white matter connectivity data. For instance, Aron et al. (2007) showed that in the stop task, a network composed of the rIFC, the pre-SMA, and the subthalamic nucleus plays a crucial role in stopping a response. In the present study, using the classical Simon task, we observed local structural differences in the anterior part of the inferior FOF. In general, the FOF has been argued to be part of the dorsal visual stream (Ungerleider and Mishkin, 1982) originating from dorsal parieto-occipital and medial parietal areas and projecting into lateral prefrontal areas (Schmahmann and Pandya, 2007). Converging evidence shows that the FOF is involved in the processing of information regarding the control of actions (Rizzolatti and Matelli, 2003). More specifically, Rizzolatti et al. (1983) suggest that the FOF may be concerned with higher-order aspects of motor behavior and the spatial aspects of attention. In a similar vein, Schmahmann and Pandya (2007) suggest that the FOF may act as a conduit by which prefrontal cortex areas may influence visual-spatial processing within the occipital and caudal parietal areas. With respect to the present data, we argue that the good inhibitors reveal a higher coherency of white matter tracts compared with poor inhibitors at an anatomical strategic site in the anterior part of the FOF. The increased coherency might give rise to an increased proficiency in implementing inhibition to overcome the incorrect visuospatial task preparation and hence the incorrect competing response in invalidly, compared with validly, cued trials. This notion was further supported by a negative correlation between the individual FA values derived from the anterior part of the FOF and the individual slope values from the slowest segment of the delta plots from the invalid cue condition (Fig. $3 B$ ). It is important to note that group analyses based on individual differences in other measures (such as the slope values from the faster delta-plot segments, the mean interference effects, or the mean overall RT) did not reveal significant local structural differences in the white matter tracts. This underlines once more the validity of exploiting the slow segments of the delta plot as a specific measure of selective response inhibition.

Our final hypothesis concerned the direct linkage between the structure and function of the rIFC and its role in implementing inhibition. A positive correlation was found between local structural differences in the anterior part of the inferior FOF and the BOLD covariation in the rIFC (Fig. 3C). Individuals revealing a strong rIFC activation in implementing inhibitory control, as shown with our model-driven fMRI analyses, also exhibited higher white matter coherency at an adjacent anatomical site. This finding links structure to function because it shows that, within the right inferior prefrontal cortex, structural individual differences in white matter connection density covary with individual differences in the proficiency of functional brain activation. Furthermore, the present results underline the cardinal importance of incorporating temporal dynamics into the analysis of response inhibition in the Simon task.

In general, this outcome attests to the power of using the combination of model-driven fMRI analysis with structural measures of the brain. In the present case, these behavioral indices were derived from RT distribution analyses that allow a focus on the efficiency of specific neurocognitive processes, typically not revealed in overall mean performance measures. By capitalizing on individual differences in these parameters (rather than relegating them to error variance), this method sheds new light on the functional and structural underpinnings of selective response inhibition in the Simon task. Note that our approach, which was 
applied here on a relatively small and homogeneous sample drawn from the normal population, holds particular promise for studying more heterogeneous samples from (sub)clinical populations suspected to be characterized by deficient impulse control, such as patients with Parkinson's disease or attention deficit/ hyperactivity disorder.

In sum, we take the present results to indicate that individual differences in behavioral indices of response inhibition predict the activity in the right IFC as well as the density of coherent white matter tracts that is necessary to suppress the initial activation and execution of the incorrect response.

\section{References}

Aron AR, Poldrack RA (2006) Cortical and subcortical contributions to Stop signal response inhibition: role of the subthalamic nucleus. J Neurosci 26:2424-2433.

Aron AR, Behrens TE, Smith S, Frank MJ, Poldrack RA (2007) Triangulating a cognitive control network using diffusion-weighted magnetic resonance imaging (MRI) and functional MRI. J Neurosci 27:3743-3752.

Beckmann CF, Jenkinson M, Smith SM (2003) General multilevel linear modeling for group analysis in fMRI. Neuroimage 20:1052-1063.

Burle B, Possamaï CA, Vidal F, Bonnet M, Hasbroucq T (2002) Executive control in the Simon effect: an electromyographic and distributional analysis. Psychol Res 66:324-336.

Daw ND, O'Doherty JP, Dayan P, Seymour B, Dolan RJ (2006) Cortical substrates for exploratory decisions in humans. Nature 441:876-879.

de Jong R, Liang CC, Lauber E (1994) Conditional and unconditional automaticity: a dual-process model of effects of spatial stimulus-response correspondence. J Exp Psychol Hum Percept Perform 20:731-750.

Eimer M (1995) Stimulus-response compatibility and automatic response activation: evidence from psychophysiological studies. J Exp Psychol Hum Percept Perform 21:837-854.

Eimer M (1999) Facilitatory and inhibitory effects of masked prime stimuli on motor activation and behavioral performance. Acta Psychol (Amst) 101:293-313.

Eriksen BA, Eriksen CW (1974) Effects of noise letters upon the identification of a target letter in a non-search task. Percept Psychophys 16:143-149.

Forstmann BU, van den Wildenberg WP, Ridderinkhof KR (2008) Neural mechansims, temporal dynamics, and individual differences in interference control. J Cogn Neurosci 20:1854-1865.

Gratton G, Coles MG, Donchin E (1992) Optimizing the use of information: strategic control of activation of responses. J Exp Psychol Gen 121:480-506

Haruno M, Kawato M (2006) Different neural correlates of reward expectation and reward expectation error in the putamen and caudate nucleus during stimulus-action reward association learning. J Neurophysiol 95:948-959.

Jenkinson M, Smith S (2001) A global optimisation method for robust affine registration of brain images. Med Image Anal 5:143-156.

Jenkinson M, Bannister P, Brady M, Smith S (2002) Improved optimization for the robust and accurate linear registration and motion correction of brain images. Neuroimage 17:825-841.

Kornblum S, Hasbroucq T, Osman A (1990) Dimensional overlap: cognitive basis for stimulus-response compatibility—a model and taxonomy. Psychol Rev 97:253-270.

Logan GD, Cowan WB (1984) On the ability to inhibit thought and action: a theory of an act of control. Psychol Rev 91:295-327.

Oldfield RC (1971) The assessment and analysis of handedness: The Edinburgh inventory. Neuropsychologia 9:97-113.

Peterson BS, Kane MJ, Alexander GM, Lacadie C, Skudlarski P, Leung HC, May J, Gore JC (2002) An event-related functional MRI study comparing interference effects in the Simon and Stroop tasks. Exp Brain Res 13:427-440.

Ridderinkhof KR (2002) Micro- and macro-adjustments of task set: activation and suppression in conflict tasks. Psychol Res 66:312-323.

Ridderinkhof KR, Scheres A, Oosterlaan J, Sergeant JA (2005) Delta plots in the study of individual differences: new tools reveal response inhibition deficits in $\mathrm{AD} / \mathrm{Hd}$ that are eliminated by methylphenidate treatment. J Abnorm Psych 114:197-215.

Rizzolatti G, Matelli M (2003) Two different streams form the dorsal visual system: anatomy and functions. Exp Brain Res 153:146-157.

Rizzolatti G, Matelli M, Pavesi G (1983) Deficits in attention and movement following the removal of postarcuate (area 6) and prearcuate (area 8) cortex in macaque monkeys. Brain 106:655-673.

Rueckert D, Sonoda LI, Hayes C, Hill DL, Leach MO, Hawkes DJ (1999) Nonrigid registration using free-form deformations: application to breast MR images. IEEE Trans Med Imaging 18:712-721.

Schmahmann JD, Pandya DN (2007) The complex history of the frontooccipital fasciculus. J Hist Neurosci 16:362-377.

Simon JR (1967) Ear preference in a simple reaction-time task. J Exp Psychol 75:49-55.

Smith SM (2002) Fast robust automated brain extraction. Hum Brain Mapp 17:143-155.

Smith SM, Jenkinson M, Johansen-Berg H, Rueckert D, Nichols TE, Mackay CE, Watkins KE, Ciccarelli O, Cader MZ, Matthews PM, Behrens TE (2006) Tract-based spatial statistics: voxelwise analysis of multi-subject diffusion data. Neuroimage 31:1487-1505.

Stroop JR (1935) Studies of interference in serial verbal reactions. J Exp Psychol 18:643-662.

Ungerleider LG, Mishkin M (1982) Two cortical visual systems. In: Analysis of visual behavior (Ingle DJ, Goodale MA, eds), pp 549-586. Cambridge, MA: MIT.

Wiegand K, Wascher E (2007) Response coding in the Simon task. Psychol Res 71:219-233.

Woolrich MW, Ripley BD, Brady M, Smith SM (2001) Temporal autocorrelation in univariate linear modeling of FMRI data. Neuroimage 14:1370-1386.

Woolrich MW, Behrens TE, Beckmann CF, Jenkinson M, Smith SM (2004) Multilevel linear modeling for fMRI group analysis using Bayesian inference. Neuroimage 21:1732-1747.

Wylie SA, Ridderinkhof KR, Eckerle MK, Manning CA (2007) Inefficient response inhibition in individuals with mild cognitive impairment. Neuropsychologia 45:1408-1419. 